\title{
Two cases of dengue meningitis: a rare first presentation
}

\author{
Rudra Prosad Goswami ${ }^{1}$, Arindam Mukherjee ${ }^{1}$, Tapan Biswas ${ }^{1}$, Partha Sarathi Karmakar ${ }^{1,2}$, \\ Alakendu Ghosh ${ }^{3}$ \\ ${ }^{1}$ Department of Internal Medicine, Institute of Post Graduate Medical Education and Research, Kolkata, West \\ Bengal, India \\ ${ }^{2}$ North Bengal Medical College and Hospital (Present), West Bengal, India \\ ${ }^{3}$ Department of Internal Medicine, Department of Clinical Immunology, Institute of Post Graduate Medical Education \\ and Research, Kolkata, West Bengal, India
}

\begin{abstract}
Dengue, a mosquito-borne disease caused by a flavivirus, is recognized in over 120 countries with 3.6 billion people living in areas at risk. Neurological manifestations are infrequently reported as clinical consequences of dengue infection. Though severe dengue may be associated with meningoencephalitis, meningitis is a rare initial presentation of otherwise uncomplicated dengue fever. We report two adult patients who presented with fever, headache, and nuchal rigidity without the typical symptoms of dengue infection. Cerebrospinal fluid (CSF) analysis showed lymphocytic pleocytosis in one and slight neutrophilic pleocytosis in the other with a normal glucose value and negative bacterial cultures. Dengue was suspected because thrombocytopenia was symptomatic in one patient and documented during the hospital course, and was confirmed by demonstration of IgM antibody in the cerebrospinal fluid samples specific for dengue in both cases. Our report demonstrates that meningitis with or without encephalitis can be the first manifestation of dengue infection. In endemic areas, dengue infection should be considered as a probable etiological agent of meningitis. Regular monitoring of platelet count can be an invaluable diagnostic screening tool. In appropriate clinical settings detection of anti-dengue IgM both in serum and in CSF may lead to correct diagnosis.
\end{abstract}

Key words: dengue; meningitis; encephalitis; neurological manifestation; CSF

J Infect Dev Ctries 2012; 6(2):208-211.

(Received 07 August 2011 - Accepted 11 December 2011)

Copyright (C) 2012 Goswami et al. This is an open-access article distributed under the Creative Commons Attribution License, which permits unrestricted use, distribution, and reproduction in any medium, provided the original work is properly cited.

\section{Introduction}

Dengue, a flavivirus disease, is recognized as one of the most important mosquito-borne human infections of the 21st century [1]. The number of cases has increased enormously, arising from more than 124 tropical and subtropical countries of the world, with 3.46 to 3.61 billion people living in areas at risk [2]. Though several neurological manifestations have been described, the true prevalence is unknown owing to underrecognition and underreporting of these rarer presentations [310]. Unlike other viral infections, meningitis determined by dengue infection is a rare complication [4]. We describe two cases of meningitis caused by oligosymptomatic dengue infection that occurred during July to August 2010 in the city of Kolkata, West Bengal, India.

\section{Case 1}

A 17-year-old woman from Sunderban, South 24 Pgs (a forest land), West Bengal, complained of a generalised, throbbing headache with neck pain. She developed fever and vomiting on the next day. She was referred to our institution on the fourth day of fever. There was no myalgia, arthralgia, bleeding manifestation, cutaneous rash or any similar or significant past medical history. Neurological examination showed the presence of nuchal rigidity, with positive Kernig's sign and Brudzinski sign. Despite the severe headache she was fully oriented to the surroundings. Initial malaria test, complete blood count, and biochemical analysis were noncontributory. Brain computed tomography (CT) scan did not show any abnormalities. Cerebrospinal fluid (CSF) was collected by lumbar puncture on the fifth day of symptoms and showed 86 leucocytes per microlitre ( $85 \%$ lymphocytes), glucose of $74 \mathrm{mg} / \mathrm{dl}$ (blood glucose $92 \mathrm{mg} / \mathrm{dl}$ ), and total protein of 116 
$\mathrm{mg} / \mathrm{dl}$. Gram and fungal stains were negative and bacterial culture was done both in CSF and in blood samples. She was being conservatively managed with intravenous hydration and ceftriaxone. On the sixth day of fever a repeat haemogram was obtained which showed thrombocytopenia (platelet count $45,000 /$ microlitre). There was no sign of bleeding tendency. We tested her blood samples for dengue infection which was positive by both NS1 antigen and presence of IgM (Dengue NS1 Ag Microlisa, J Mitra and Co, New Delhi, India and IVD Microwell Dengue Fever Kit, IVD Research Inc, Carlsbad, CA USA, respectively). To further strengthen the diagnosis, we performed a repeat CSF aspiration to test for IgM antibodies against dengue, Japanese encephalitis (IgM monoclonal antibody-capture ELISA; National Institute of Virology, Pune, India) and HSV-1. Her CSF sample was positive for dengue IgM only. By this time the cultures were available and were negative. We withdrew the intravenous antibiotics and put her on oral analgesics and intravenous hydration for two days followed by oral hydration, on which she recovered completely by the tenth day of fever. She did well on follow-up and a repeat examination of IgM against dengue four weeks after the initial examination showed a six-fold rise using the same kit.

\section{Case 2}

A 42-year-old male patient from rural Murshidabad, West Bengal, was admitted with clouding of consciousness, high-grade continuous fever with chills, and headache for three days without history of cough or coryza, dysuria, abdomen pain, skin rash or any episode of convulsion or similar or significant past medical history.

On physical examination he was found confused (Glasgow Coma Scale of 8) and febrile (surface temperature of $40^{\circ} \mathrm{C}$ ) with severe neck rigidity and bilateral extensor plantar responses and with ankle clonus. He was haemodynamically stable.

Possible differential diagnoses were meningoencephalistis (viral, bacterial or remotely mycobacterial), cerebral malaria, brainstem or subarachnoid hemorrhage, or generalised sepsis with encephalopathy. The CT scan of the brain was normal. Both malaria parasite and dual antigen tests were negative. Blood biochemistry revealed normal electrolytes $\left(\mathrm{Na}^{+} 137.9 \mathrm{mmol} / \mathrm{L} ; \mathrm{K}^{+} 4.53 \mathrm{mmol} / \mathrm{L}\right.$; $\left.\mathrm{Ca}^{++} 8.5 \mathrm{mg} / \mathrm{dl}\right)$, renal function and raised liver enzymes (SGOT 169; SGPT 363). Complete haemogram was within normal limits. The CSF study showed 10 leucocytes per microlitre $(70 \%$ neutrophils), glucose of $77 \mathrm{mg} / \mathrm{dl}$ (blood glucose 97 $\mathrm{mg} / \mathrm{dl}$ ), total protein of $41 \mathrm{mg} / \mathrm{dl}$, and adenosine deaminase level of $0.5 \mathrm{U} / \mathrm{L}$. Gram and fungal stains were negative and bacterial culture was performed both in CSF and in blood samples. We started treatment with nasogastric feeding, intravenous ceftriaxone, acyclovir, and vancomycin empirically. The patient was showing some signs of improvement until the third day of admission when he developed repeated bouts of malaena. A haemogram revealed thrombocytopenia $(30,000$ per microitre). Though acyclovir induced thrombocytopenia and ceftriaxone induced platelet dysfunction could not be ruled out, we considered dengue as a possible aetiology. Dengue IgM (IVD Microwell Dengue Fever Kit, IVD Research Inc, Carlsbad, CA, USA) was positive in serum on the fourth day of admission and the seventh day of fever. We reaspirated CSF and tested for dengue IgM, and the test result was positive. The culture reports came negative the next day. CSF and blood samples for detection of IgM antibodies against Japanese encephalitis virus or HSV-1 were also negative. We withdrew all antibiotics and the antiviral, but continued fluid resuscitation and paracetamol. The patient regained consciousness and was afebrile by the tenth day of admission. He was successfully discharged without any neurological complications on the $14^{\text {th }}$ day of admission. He did well on follow-up and repeat titres of $\operatorname{IgM}$ against dengue, 5 weeks after the initial examination, showed a five-fold rise using the same kit.

\section{Discussion}

Our cases occurred during the rainy seasons of 2010 and did not share the typical symptomatology of dengue. There was no history of myalgia, arthralgia, retro-orbital pain, petechiae or purpura, thrombocytopenia, rash, or bleeding at presentation. The main symptoms were severe headache and fever with the main clinical finding of nuchal rigidity bringing the cases under the spectrum of meningoencephalitis depending on the presence of altered sensorium. The first case was meningitis without any encephalitic component. The second one was meningoencephalitis. Our final diagnosis was neurological manifestation as the first presentation of dengue fever in both the instances.

Dengue is a rare cause of viral meningitis [4]. Encephalitis is a more recognised feature. In a prospective study by Soares et al. from Brazil, dengue emerged as the leading cause of encephalitis 
with normal CSF cellularity (75\%). On the other hand, dengue was found in only a minority of patients presenting mainly with meningitis [5]. A Jamaican study found $13.5 \%$ of dengue patients presenting with neurological symptoms or signs with encephalitis as the leading feature. Meningitis due to dengue was present in only $4 \%$ (18 out of 401) of patients [6]. Other neurological manifestations of dengue include transverse myelitis [7], Guilliain-Barré syndrome [8], and acute disseminated encephalomyelitis [9]. Encephalitis is thought to occur in up to $6.2 \%$ of patients with dengue haemorrhagic fever but is rare in dengue fever [10].

Time of onset of neurological symptoms in dengue has been reported to range from three to seven days on an average from the start of fever [10]. In our first case, headache and neck pain occurred earlier than fever onset. In the second case, fever and neurological symptoms were concomitant at onset.

In both the cases the diagnosis was suspected after demonstration of thrombocytopenia, accidentally in the first instance and owing to bleeding manifestation in the second, which was most prominent on the sixth day of fever. This observation is in good agreement with a large Brazilian study that included 543 dengue patients and found a decrease in platelet count from the third day of fever in uncomplicated cases, while thrombocytopenia started from one and two days in dengue haemorrhagic fever. In both groups the lowest platelet count occurred around the seventh day of fever [11].

Both our cases recovered completely from the ailments and they were followed up for one month without any residual neurodeficit. Misra et al. found that recovery was complicated with three out of 11 dying patients and another three with residual neurodeficit [12]. On the other hand Kankirawatana et al. [13], Solomon et al. [7], and Kularatne et al. [14] have shown a more benign outcome.

Severe dengue is defined by one or more of the following: (i) plasma leakage that may lead to shock (dengue shock) and/or fluid accumulation, with or without respiratory distress; and/or (ii) severe bleeding; and/or (iii) severe organ impairment. Only two terms are of neurological significance are compounded in the WHO guideline concerning severe organ impairment, namely encephalopathy and encephalitis [15]. The case definition of dengue encephalitis has been recently propounded, which includes presence of fever, headache, reduced consciousness level, unexplained acute liver failure, shock, electrolyte derangement or intracranial haemorrhage with detection of dengue virus or $\operatorname{IgM}$ in the serum or CSF, and suggestive neuroimaging features [10]. In most cases diagnosis depends on detection of the virus itself (by culture, PCR, or NS1 antigen detection) or detection of host immune reaction (IgM type antibody capture, MAC ELISA, for anti dengue IgM or haemagglutination inhibition test). The gold-standard method for viral detection has traditionally been viral culture, although it is difficult and time-consuming. PCR assays are quicker, allow discrimination between viral serotypes, and have been shown to be highly specific within the first five days of fever [16]. PCR assays have recently been utilised in a recent report by Soares et al. in the presentation of a case of pure oligosymtomatic dengue meningitis in 2010, in which type 3 dengue virus was isolated as the pathogen [3]. Dussart et al. have achieved a sensitivity of $89 \%$ with an assay for NS1 antigen [17]. This test is rapid, reliable and less costly than PCR. In a recent study in fatal dengue meningoencephalitis cases, a kit for detection of the NS1 antigen in CSF gave sensitivity of $50 \%$ and specificity of $100 \%$. When used in combination with $\mathrm{IgM}$, the detection rate rose to $92.3 \%$ [18]. Another recent study by Puccioni-Sohler [19] evaluated CSF and serum dengue $\operatorname{IgM}$ and $\operatorname{IgG}$ in dengue patients with neurological manifestations; their results showed that 7 out of 10 of their patients had evidence of intrathecal production of $\operatorname{IgM}$ in response to dengue infection. This observation also lays support to test for dengue specific IgM in CSF in patients with neurological manifestations in the background of dengue infection [19].

MAC ELISA measures dengue-specific IgM. One time presence of anti-dengue antibodies vouches for recent infection (within 24 weeks). A rising titre in two serum samples can confirm acute infection. Singh et al. reported the sensitivity of MAC-ELISA at $69 \%$, rising to $90 \%$ with repeat convalescent testing. Specificity was $80 \%$ [15].

It is generally recommended to use PCR or NS1 antigen detection in patients with fever for fewer than five days, and MAC ELISA in patients with fever for more than five days [10]. In the clinical spectrum of viral meningoencephalitis, especially when dengue is a possibility, other flaviviruses such as Japanese encephalitis, which is endemic in India, should be excluded. A recent report from Assam also indicates that West Nile virus circulates in the Indian environment [20]. How much contribution of acute viral meningoencephalitis can be laid on West Nile 
virus remains to be elucidated. As already mentioned, a four-fold or greater rise of antibody titres on paired serum samples should be performed to confirm the diagnosis, as was done in our case.

\section{Conclusion}

Our report demonstrates that meningitis with or without encephalitis can be the first manifestation of dengue infection. In endemic areas, dengue infection should be considered as a probable etiological agent of meningitis. Regular monitoring of platelet count can be an invaluable diagnostic screening tool. If indicated, CSF examination for dengue IgM should be performed.

\section{Acknowledgements}

We acknowledge the essential role of the paramedical staff in our institution in providing the proper care for these patients, which inspired us to have adequate insight into the patients' ailments. We also acknowledge the Head of the Department of Internal Medicine, Professor Tapash Das, for his encouragement to pursue the cases and get them published.

\section{Consent}

Written informed consent was obtained from the patients for publication of this Case Report. A copy of the written consent is available for review by the Editor-in-Chief of this journal.

\section{References}

1. Dash PK, Parida MM, Saxena P, Abhyankar A, Singh CP, Tewari KN, Jana AM, Sekhar K, Rao PV (2006) Reemergence of dengue virus type-3 (subtype-III) in India: implications for increased incidence of DHF/DSS. Virol J 3: 55.

2. Lim KW, Sit NW, Norzahira R, Sing KW, Wong HM, Chew HS, Firdaus R, Cheryl JA, Suria M, Mahathavan M, Nazni WA, Lee HL, McKemy A, Vasan SS (2010) Dengue vector surveillance in insular settlements of Pulau Ketam, Selangor, Malaysia. Trop Biomed 27: 185-192.

3. Soares CN, Cabral-Castro MJ, Peralta JM, Freitas MR, Puccioni-Sohler M (2010) Meningitis determined by oligosymptomatic dengue virus type 3 infection: report of a case. Int J Infect Dis 14: 150-152.

4. Chadwick DR (2006) Viral meningitis. Br Med Bull 75-76: $1-14$.

5. Soares CN, Cabral-Castro MJ, Peralta JM, de Freitas MR, Zalis M, Puccioni-Sohler M (2011) Review of the etiologies of viral meningitis and encephalitis in a dengue endemic region. J Neurol Sci 303: 75-79.

6. Jackson ST, Mullings A, Bennett F, Khan C, GordonStrachan G, Rhoden T (2008) Dengue infection in patients presenting with neurological manifestations in a dengue endemic population. West Indian Med J 57: 373-376.

7. Solomon T, Dung NM, Vaughn DW, Kneen R, Thao LT, Raengsakulrach B, Loan HT, Day NP, Farrar J, Myint KS, Warrell MJ, James WS, Nisalak A, White NJ (2000) Neurological manifestations of dengue infection. Lancet 355: 1051053-9.

8. Sulekha C, Kumar S, Philip J (2004) Guillain-Barre syndrome following dengue fever. Indian Pediatr 41: 948950.
9. Yamamoto $\mathrm{Y}$, Takasaki T, Yamada K, Kimura M, Washizaki K, Yoshikawa K, Hitani A, Nakamura T, Iwamoto A (2002) Acute disseminated encephalomyelitis following dengue fever. J Infect Chemother 8: 175-177.

10. Varatharaj A (2010) Encephalitis in the clinical spectrum of dengue infection. Neurol India 58: 585-591.

11. Oliveira EC, Pontes ER, Cunha RV, Fróes IB, Nascimento D (2009) Hematological abnormalities in patients with dengue. Rev Soc Bras Med Trop 42: 682-685.

12. Misra UK, Kalita J, Syam UK, Dhole TN (2006) Neurological manifestations of dengue virus infection. J Neurol Sci 244: 117-122.

13. Kankirawatana $P$, Chokephaibulkit K, Puthavathana $P$, Yoksan S, Somchai A, Pongthapisit V (2000) Dengue infection presenting with central nervous system manifestation. J Child Neurol 15: 544-547.

14. Kularatne SA, Pathirage MM, Gunasena S (2008) A case series of dengue fever with altered consciousness and electroencephalogram changes in Sri Lanka. Trans R Soc Trop Med Hyg 102: 1053-1054.

15. Alexander N, Balmaseda A, Coelho IC, Dimaano E, Hien TT, Hung NT, Jänisch T, Kroeger A, Lum LC, Martinez E, Siqueira JB, Thuy TT, Villalobos I, Villegas E, Wills B; on behalf of the European Union, World Health Organization (WHO-TDR) supported DENCO Study Group (2011) Multicentre prospective study on dengue classification in four South-east Asian and three Latin American countries. Trop Med Int Health. May 30. doi: 10.1111/j.13653156.2011.02793.x. [Epub ahead of print]

16. Singh K, Lale A, Ooi EE, Chiu L-L, Chow VTK, Tambyah P, E, Koay ESC (2006) A prospective clinical study on the use of reverse transcription-polymerase chain reaction for the early diagnosis of dengue fever. J Mole Diagn 8: 613616.

17. Dussart P, Labeau B, Lagathu G, Louis P, Nunes MR, Rodrigues SG, Storck-Herrmann C, Cesaire R, Morvan J, Flamand M, Baril L (2006). Evaluation of an enzyme immunoassay for detection of dengue virus NS1 antigen in human serum. Clin Vacc Immunol 13: 1185-1189.

18. Araújo FM, Brilhante RS, Cavalcanti LP, Rocha MF, Cordeiro RA, Perdigão AC, Miralles IS, Araújo LC, Araújo RM, Lima EG, Sidrim JJ (2011) Detection of the dengue non-structural 1 antigen in cerebral spinal fluid samples using a commercially available enzyme-linked immunosorbent assay. J Virol Methods 177: 128-131.

19. Puccioni-Sohler M, Soares CN, Papaiz-Alvarenga R, Castro MJ, Faria LC, Peralta JM (2009) Neurologic dengue manifestations associated with intrathecal specific immune response. Neurology 73: 1413-1417.

20. Khan SA, Dutta P, Khan AM, Chowdhury P, Borah J, Doloi P, Mahanta J (2011) West Nile virus infection, Assam, India. Emerg Infect Dis 17: 947-948.

\section{Corresponding author}

Rudra Prosad Goswami

Department of Internal Medicine

Institute of Post Graduate Medical Education and Research

Kolkata, West Bengal

Telephone: +919433534335

Email: rudra.goswami@gmail.com

Conflict of interests: No conflict of interests is declared. 